\title{
Pre-Endoscopy Drink of Simethicone and N-Acetylcysteine Significantly Improves Visualization in Upper Gastrointestinal Endoscopy
}

\author{
Shrihari Anil Anikhindi ${ }^{1}$ Ashish Kumar ${ }^{1} \quad$ Noriya Uedo ${ }^{2}$ Vikas Singla ${ }^{1}$ Akshay Anikhindi ${ }^{3}$ \\ Praveen Sharma ${ }^{1}$ Naresh Bansal ${ }^{1}$ Piyush Ranjan ${ }^{1} \quad$ Mandhir Kumar $^{1}$ Munish Sachdeva ${ }^{1}$ \\ Shivam Khare ${ }^{1}$ Anil Arora ${ }^{1}$
}

${ }^{1}$ Institute of Liver, Gastroenterology and Pancreaticobiliary Sciences, Sir Ganga Ram Hospital, New Delhi, India

${ }^{2}$ Department of Gastrointestinal Oncology, Osaka International Cancer Institute, Osaka, Japan

${ }^{3}$ Pravara Institute of Medical Sciences, Loni, Maharashtra, India

\begin{abstract}
Address for correspondence Shrihari Anil Anikhindi, MD, DNB, Institute of Liver, Gastroenterology and Pancreaticobiliary Sciences, Sir Ganga Ram Hospital, Rajinder Nagar, New Delhi 110060, India (e-mail: dr.anikhindi@gmail.com).
\end{abstract}

J Digest Endosc 2021;12:11-18.

\author{
Abstract \\ Keywords \\ - endoscopic mucosal \\ resection \\ - endoscopic \\ submucosal dissection \\ - mucosal visibility \\ - N-acetylcysteine \\ - narrow band imaging \\ - pre-endoscopy \\ preparation \\ - simethicone
}

Introduction With the advancements in diagnostic and therapeutic upper gastrointestinal endoscopy (UGIE), clear mucosal visualization is essential to ensure optimal outcomes. Though routinely followed in Japan and Korea, pre-endoscopic preparation is seldom used in India. We evaluated the efficacy of a pre-endoscopic drink of $\mathrm{N}$-acetylcysteine (NAC) and simethicone in improving mucosal visibility during UGIE.

Patients and Methods This study was a retrospective, investigator blind study with a case-control study design. Cases included patients who received a pre-endoscopy drink of NAC and simethicone in $100 \mathrm{~mL}$ water administered 10 to 30 minutes prior to UGIE. Controls only had mandatory fasting for 6 to 8 hours prior to UGIE. Propensity score matching was done to ensure comparability between the groups. Digital images were taken at six standard landmarks during UGIE and stored. A blinded investigator subsequently analyzed the images and rated the mucosal visibility on a 3-point scale. The difference in the mean mucosal visibility between the cases and controls was compared.

Results Mean mucosal visibility during UGIE was significantly better using NAC with simethicone as compared with no preparation at esophagus (1.14 [0.37] vs. 1.47 [0.62], $p<0.05)$, gastric fundus (1.10 [0.30] vs. 1.55 [0.64], $p<0.05)$, gastric body (1.22 [0.50] vs. 1.62 [0.73], $p<0.05)$, gastric antrum (1.13 [0.37] vs. 1.47 [0.62], $p<0.05)$, and duodenal bulb (1.13 [0.34] vs. $1.33[0.56], p<0.05)$. In distal duodenum, though visibility improved with NAC with simethicone, the difference was insignificant. There were no adverse events related to the pre-endoscopy drink.

Conclusion A pre-endoscopy drink of NAC with simethicone can significantly improve mucosal visibility during UGIE. It is safe, cheap, easily available and maybe considered for routine utilization for ensuring optimal endoscopic outcomes. published online October 17, 2020
DOI https://doi.org/ $10.1055 / \mathrm{s}-0041-1726225$ ISSN 0976-5042.
(O2020. Society of Gastrointestinal Endoscopy of India.

This is an open access article published by Thieme under the terms of the Creative Commons Attribution-NonDerivative-NonCommercial-License, permitting copying and reproduction so long as the original work is given appropriate credit. Contents may not be used for commercial purposes, or adapted, remixed, transformed or built upon. (https://creativecommons.org/licenses/by-nc-nd/4.0/). Thieme Medical and Scientific Publishers Pvt. Ltd. A-12, 2nd Floor, Sector 2, Noida-201301 UP, India 


\section{Introduction}

The realms of diagnostic and therapeutic endoscopy have rapidly expanded in past few decades. Many endoscopy centers, especially in India, have recently started focusing on advanced endoscopic modalities like narrow band imaging (NBI), endoscopic mucosal resection (EMR), and endoscopic submucosal dissection (ESD). Good pre-endoscopy preparation is essential before commencing these procedures for unhindered and detailed evaluation of gastrointestinal mucosa.

Pre-procedural preparation prior to colonoscopy has become a norm world over and several bowel preparation scores have been validated for use in clinical practice. ${ }^{1}$ However, there is no standardized preparation recommended prior to upper gastrointestinal endoscopy (UGIE). In most centers in India and around the world, the only requirement is fasting for 6 to 8 hours prior to UGIE. ${ }^{2}$ A recently published European Society of Gastrointestinal Endoscopy (ESGE) Quality Improvement Initiative also mentioned only fasting prior to UGIE as a key performance measure. ${ }^{3}$

In routine endoscopy practice, it is frequently observed that in fasting state foam and mucus frequently impair mucosal visualization during UGIE. Pioneering endoscopy centers, especially in Japan and Korea, routinely use a pre-endoscopy preparation. ${ }^{4}$ Most of the preparations used consist of a defoaming agent like simethicone with or without a proteolytic agent like pronase or $\mathrm{N}$-acetylcysteine (NAC). ${ }^{5}$ Simethicone acts by reducing the surface tension of the air bubbles, thus coalescing and dispersing them without absorption in the gastrointestinal mucosa. ${ }^{6}$ Pronase is a proteolytic enzyme that aids in mucolysis; but is not available in India. NAC can be used as an alternative to pronase.

Studies on efficacy of pre-endoscopy preparations have been predominantly published from East Asia or the West. ${ }^{7,8,9,10,11,12}$ These studies have consistently showed significant improvement in mucosal visibility along with reduction in UGIE procedure time and reduced need of intraprocedural flushing.

Average number of endoscopies performed by each endoscopist in average Indian centers is usually higher as compared with West or Japan and Korea. Having good endoscopic pre-preparation is a desirable attribute as it would not only be a stepping stone to venture into advanced endoscopic diagnosis and therapy but also reduce procedure times and effort. There are no published studies from India except one abstract that have explored the outcomes of pre-endoscopy preparation. ${ }^{13}$

We recently started the protocol of administering pre-endoscopy preparation to all patients posted for UGIE. In the present study, we compared the effect of administering a pre-endoscopy drink having a combination of simethicone and NAC in terms of improving mucosal visibility during UGIE with UGIE without this preparation.

\section{Patients and Methods}

\section{Study Setting}

This study was a retrospective, investigator blind study with a case-control study design, conducted in the Institute of
Liver, Gastroenterology and Pancreaticobiliary Sciences at Sir Ganga Ram Hospital, New Delhi, India. Being a retrospective study, ethical committee approval was not required. The primary objective was to evaluate whether a pre-endoscopic drink consisting of simethicone and NAC with water (NAC + simethicone) improved mucosal visibility during UGIE as compared with no pre-endoscopy preparation. Informed consent was obtained from each patient before the UGIE both for the procedure and for inclusion of their data for research study.

\section{Patients}

We started the protocol of administering pre-endoscopy preparation to most patients posted for UGIE since January 2020. Prior to this period, all UGIE procedures were done only with mandatory fasting (including no water intake) for a period of 6 to 8 hours prior to the procedure.

For our study, all consecutive patients who received pre-endoscopy preparation from January 2020 through March 2020 were taken as cases. Equal number of propensity score matched patients were taken as controls who had undergone UGIE between October 2019 through December 2019, without pre-endoscopy preparation, but only with mandatory fasting.

Patients with following indications for UGIE were included in the study: dyspepsia, gastroesophageal reflux disease, chronic liver disease for evaluation of portal hypertension related changes, anemia, unexplained weight loss, chronic diarrhea, and recurrent vomiting.

Following patients were excluded from our study: Patients with acute ( $<48$ hours) or ongoing upper gastrointestinal bleeding, pregnant or lactating patients, known hypersensitivity to simethicone or NAC, esophageal stricture or gastric outlet obstruction or any other anatomical cause precluding complete endoscopic evaluation up to second part of duodenum, patients with altered anatomy due to upper gastrointestinal surgery, severe cardiorespiratory illness, those with retained food residue in stomach due to inadequate fasting or otherwise, severe esophageal or gastric inflammation due to corrosive intake/viral/fungal etiology, etc., and those unwilling to provide informed written consent for inclusion in study.

\section{Pre-Endoscopy Drink}

Cases were administered a pre-endoscopy drink between 10 and 30 minutes prior to undergoing UGIE. Each drink contained:

- $100 \mathrm{~mL}$ drinking water

- One tablet of NAC flavored effervescent tablet, $600 \mathrm{mg}$ Mucotab ET German Remedies, a unit of Cadila Healthcare Limited, Gujarat, India

- One milliliter of simethicone emulsion containing $40 \mathrm{mg}$ simethicone (Medigas, Rapross Pharmaceuticals Pvt. Ltd., New Delhi, India)

\section{Endoscopy Procedures}

UGIE procedures were performed by experienced endoscopists (SAA and VS) each having performed more than 5000 UGIE procedures prior to onset of study. UGIE was done 
with Olympus 180 (Evis Exera II GIF $180 \mathrm{H}$ ) or 190 series (Evis Exera III, GIF 190 HQ) (Olympus Medical, Tokyo, Japan). All UGIE procedures were done under mild sedation with 2 or $3 \mathrm{mg}$ of intravenous midazolam.

After intubation of stomach and duodenum, excess fluid encountered if any was aspirated. Electronic photographs were captured as per standard reporting followed by our department at six predefined locations lower esophagus, gastric fundus, mid gastric body, gastric antrum, duodenal bulb, and second part of duodenum. For the purpose of study, in cases, the images were captured in native mucosal state before using a water flush. Images were stored digitally (Endo Clinic, Ottomed Endoscopy) for future mucosal visibility scoring by an independent blinded investigator (AK).

\section{Mucosal Visibility Scoring}

Set of digital images of UGIE of cases and controls were provided to a blinded investigator (AK). He was not part of any of the UGIE procedure. The investigator was asked to mark the mucosal visibility on a 3-point scale at six endoscopic sites as follows:

1. Excellent-clear visibility of the mucosa with no bubbles or mucus (-Fig. 1).

2. Suboptimal-small amount of bubbles or mucus, not obscuring mucosal vision (-Fig. 2).

3. Poor-significant amount of bubbles or mucus, obscuring mucosal vision (-Fig. 3).

The mean mucosal visibility score at each of the six sites for cases and controls was compared. Also, proportion of patients having excellent mucosal visibility was compared in the two groups.

\section{Primary Objective}

The primary objective was to see the change in mean mucosal visibility scores in UGIE rated by blinded investigator after administration of a pre-endoscopy drink containing simethicone and NAC.

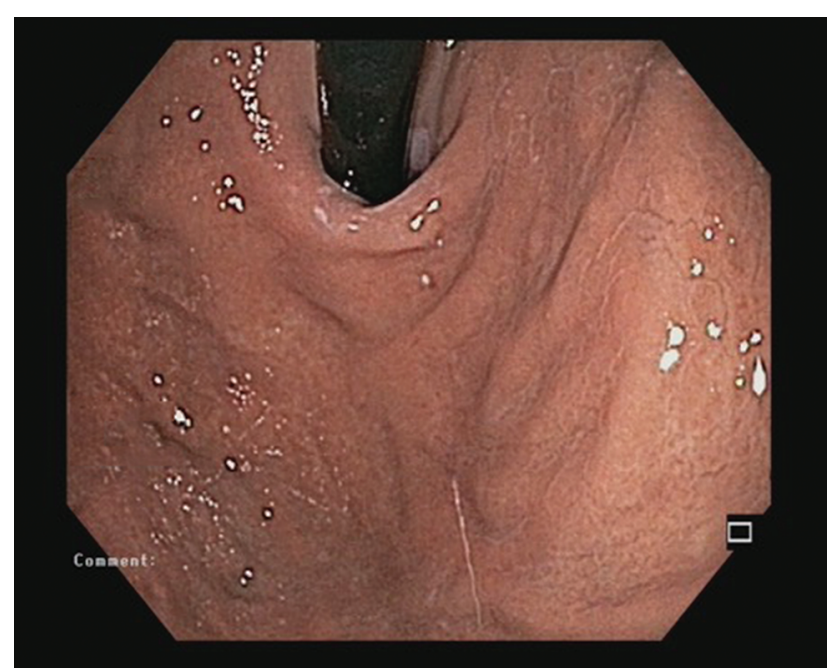

Fig. 1 Retroversion view of gastric fundus. Mucosal visibility score 1 (excellent) with no bubbles or mucus.

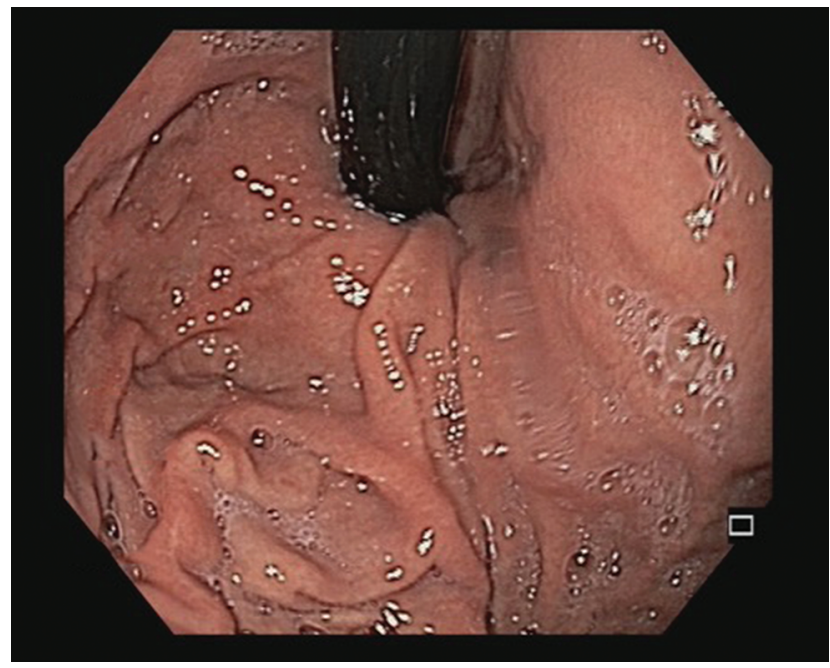

Fig. 2 Retroversion view of gastric fundus. Mucosal visibility score 2 (sub optimal) with small amount of bubbles or mucus, not obscuring mucosal vision.

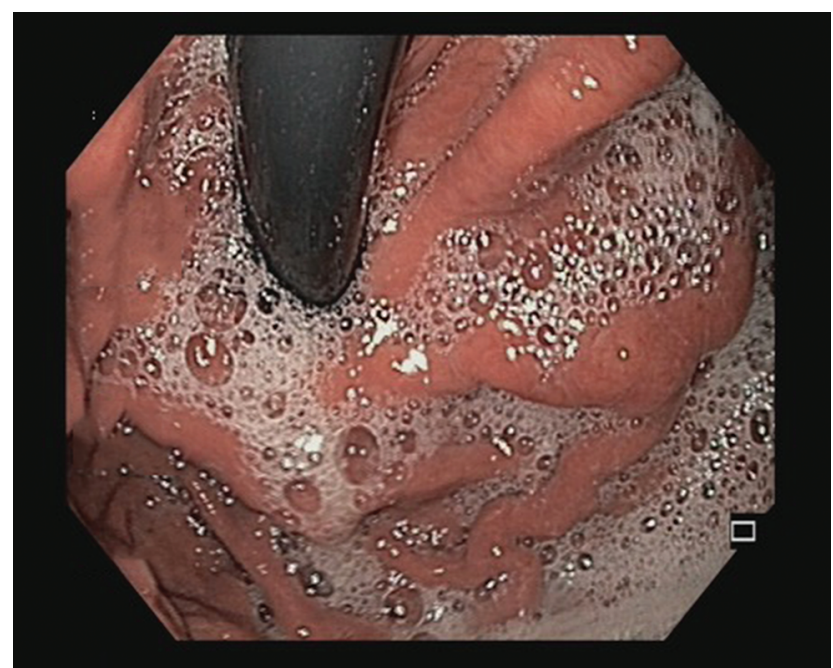

Fig. 3 Retroversion view of gastric fundus. Mucosal visibility score 3 (poor) with significant amount of bubbles or mucus, obscuring mucosal vision.

\section{Statistics}

Sample size calculation was derived considering the proportion of patients with excellent mucosal visibility during UGIE being $70 \%$ as seen in previous study. ${ }^{8}$ We expected a $20 \%$ improvement in excellent visibility after pre-endoscopic drink of NAC + simethicone (i.e., $90 \%$ excellent visibility proportion). Taking the power of study as $80 \%$ and $\alpha$ error of 0.05 , we got the minimum required sample size as 124 (62 in each group).

Propensity score matching of controls with cases was done using age, gender, and indications for UGIE as covariates. Statistician involved in propensity score matching had no access to the images or reports of the endoscopies.

Continuous variables were expressed as mean with standard deviation and discrete variables were expressed as number (\%). Comparison of continuous variables between two groups was done using Student's t-test. Mann-Whitney $\mathrm{U}$ test was used to compare ranks (mucosal visibility scores). 
14 Pre-Endoscopy Simethicone and N-Acetylcysteine Anikhindi et al.

Fisher's exact test or chi-squared test was used to compare categorical variables. SPSS 20 (Chicago, Illinois, United States) software was used for analysis.

\section{Results}

\section{Enrolment and Exclusions}

During the case enrolment period, total 244 UGIE were done in the department. After exclusions as per protocol and propensity score matching, 108 patients were enrolled as cases for receiving the pre-endoscopy drink. After UGIE was performed, four additional patients were excluded ( - Fig. 4). Two patients had severe esophagitis precluding good mucosal survey, one patient had food residue in stomach despite adequate fasting and was later diagnosed to have gastroparesis, another patient had no obvious history of gastrointestinal bleed but showed altered blood in the stomach from a Mallory-Weiss tear.

For the control group, patients having undergone UGIE between October 2019 and December 2019 were considered. Total 291 UGIE procedures were performed during this period. Among these, 108 controls were selected after considering the exclusion criteria and performing the propensity score matching. While evaluating the endoscopic images, two additional patients were excluded due to incomplete endoscopic report in one patient (absence of photographs from one or more predefined landmarks) and evidence of food residue in the stomach on the endoscopic images in one patient ( - Fig. 4).

\section{Demography}

Both the study groups were matched for mean age (47 vs. 49 years, $p=0.43$ ), sex distribution (66 vs. 57\% males, $p=$ 0.20 ), and indications for UGIE. Most common indication for UGIE in was dyspepsia (31 and 34\%), gastroesophageal reflux disease (19 and 16\%), and screening or follow-up of patients with chronic liver disease (17 and 19\%) (-Table 1).

\section{Mucosal Visibility Scores}

When evaluated for the primary objective of mucosal visibility score, there was significant difference between the two groups at most landmarks. The mean mucosal visibility score in patients who received a pre-endoscopic drink of simethicone with NAC was much better in the esophagus (1.14 [0.37] vs. 1.47 [0.62], $p<0.001)$, gastric fundus $(1.10$ [0.30] vs. 1.55 [0.64], $p<0.001)$, gastric body (1.22 [0.50] vs.

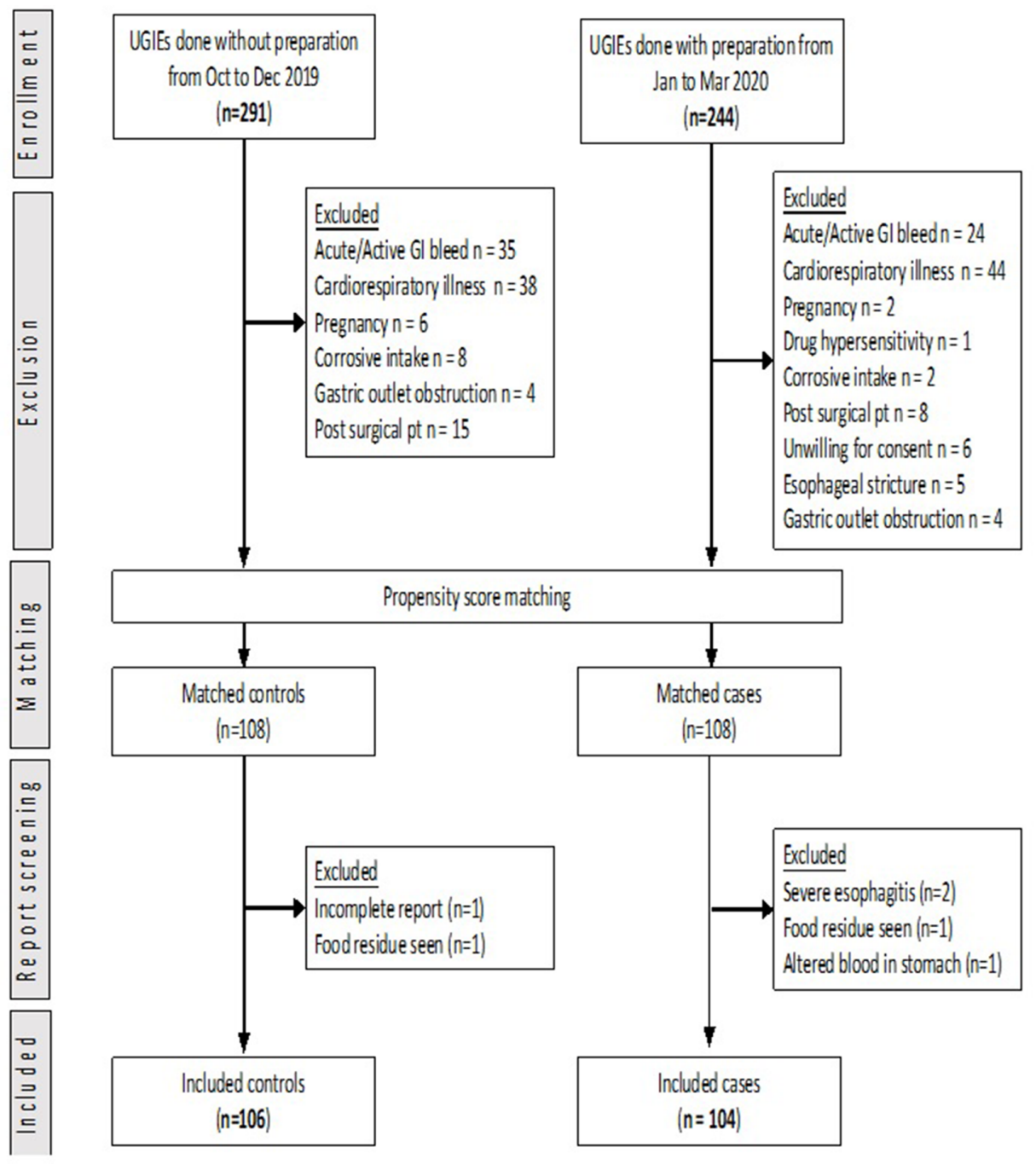

Fig. 4 Patient enrollment flowchart. 
1.62 [0.73], $p<0.001)$, gastric antrum (1.13 [0.37] vs 1.47 [0.62], $p<0.001)$, and duodenal bulb (1.13 [0.34] vs. 1.33 [0.56], $p<0.001$ ) ( Table 2).

However, when seen in second part of duodenum, though mucosal visibility score was better with the pre-endoscopy drink, the difference was not statistically significant (1.17 [0.38] vs. 1.28 [0.49], $p=0.10$ ).

\section{Percentage of Patients with "Excellent Preparation"}

A score of 1 that meant clear visibility of mucosa with no presence of any bubbles or mucous was considered excellent as this is the ideal requirement for a good UGIE. Patients who had received the pre-endoscopy drink of simethicone with NAC had a significantly higher percentage of excellent endoscopic preparations in the esophagus (87 vs. $60 \%, p<0.001$ ), gastric fundus (90 vs. 53\%, $p<0.001$ ), gastric body (82 vs. $53 \%, p<0.001$ ), gastric antrum (88 vs. $60 \%, p<0.001$ ), and duodenal bulb (87 vs. $72 \%, p<0.001$ ) (-Table 3, - Fig. 5).

Percentage of patients with excellent visibility after pre-endoscopic drink was also higher in second part of duodenum, but the difference did not assume significance ( 83 vs. $74 \%, p=0.07)$.

Table 1 Demography

\begin{tabular}{|c|c|c|c|}
\hline & $\begin{array}{l}\text { No preparation } \\
(n=106)\end{array}$ & $\begin{array}{l}\text { NAC + simethicone } \\
(n=104)\end{array}$ & $p$-Value \\
\hline Age, mean (SD), years & $47(16)$ & $49(16)$ & 0.43 \\
\hline \multicolumn{4}{|l|}{ Sex, $n(\%)$} \\
\hline Male & $70(66)$ & $59(57)$ & \multirow[t]{2}{*}{0.20} \\
\hline Female & $36(34)$ & $45(43)$ & \\
\hline \multicolumn{4}{|l|}{ Indication for procedure, $n(\%)$} \\
\hline Dyspepsia & $33(31)$ & $35(34)$ & \multirow[t]{6}{*}{0.94} \\
\hline GERD & 20 (19) & $17(16)$ & \\
\hline CLD & $18(17)$ & $20(19)$ & \\
\hline Anemia & $14(13)$ & $11(11)$ & \\
\hline Weight loss & $9(9)$ & $7(7)$ & \\
\hline Others & $12(11)$ & $14(14)$ & \\
\hline
\end{tabular}

Abbreviations: CLD, chronic liver disease; GERD, gastroesophageal reflux disease; NAC, N-acetylcysteine; SD, standard deviation.

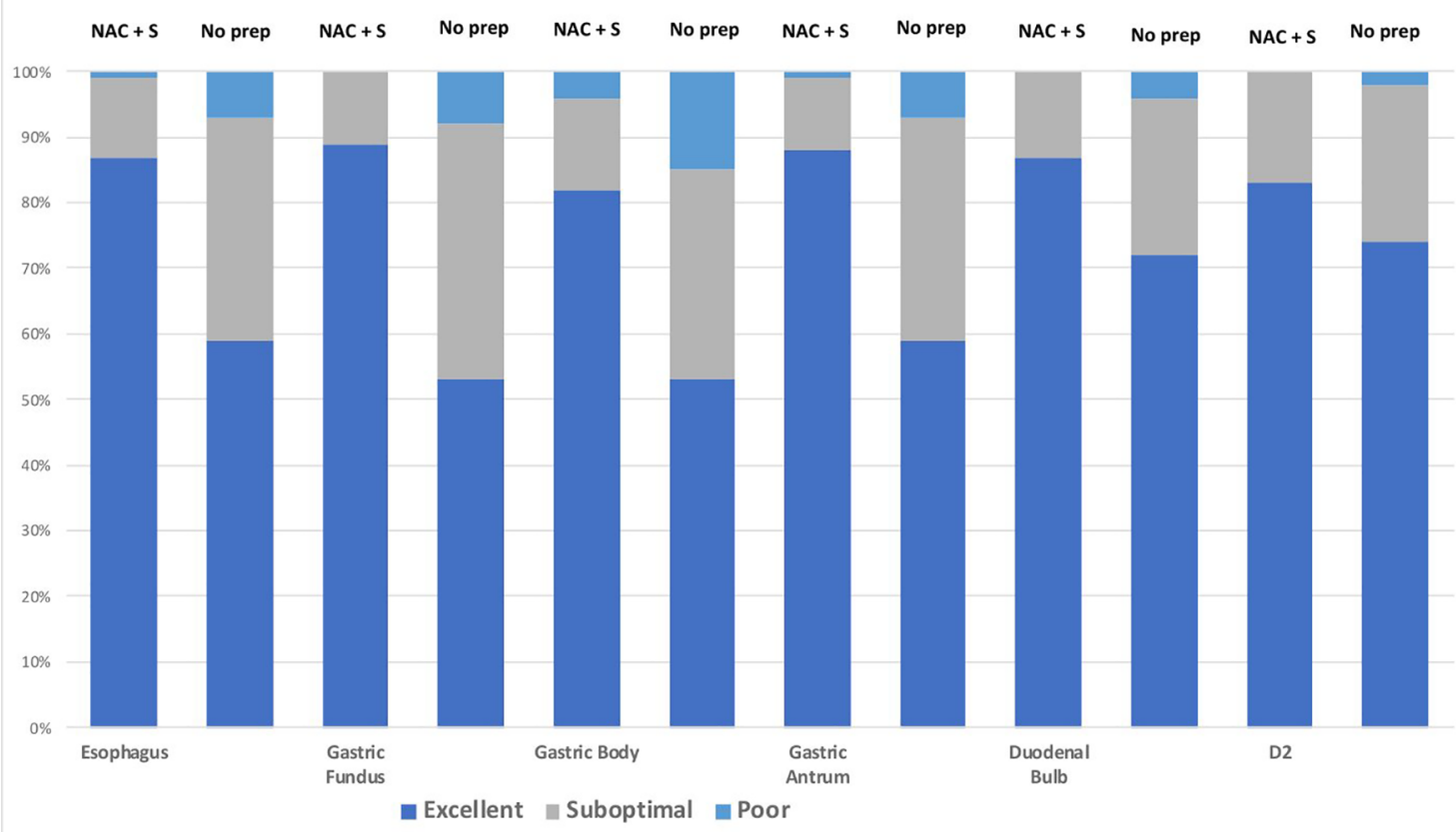

$\mathrm{NAC}+\mathrm{S}=\mathrm{N}$-acetylcysteine with Simethicone

No prep $=$ No preparation

Fig. 5 Effect of pre-endoscopy drink on endoscopic visibility. 
Table 2 Mucosal visibility score

\begin{tabular}{|c|c|c|c|}
\hline & $\begin{array}{l}\text { No preparation } \\
(n=106)\end{array}$ & $\begin{array}{l}\text { NAC + simethicone } \\
(n=104)\end{array}$ & $p$-Value \\
\hline Esophagus, mean \pm SD & $1.47(0.62)$ & $1.14(0.37)$ & $<0.001$ \\
\hline \multicolumn{4}{|l|}{ Stomach, mean \pm SD } \\
\hline Fundus & $1.55(0.64)$ & $1.10(0.30)$ & $<0.001$ \\
\hline Body & $1.62(0.73)$ & $1.22(0.50)$ & $<0.001$ \\
\hline Antrum & $1.47(0.62)$ & $1.13(0.37)$ & $<0.001$ \\
\hline \multicolumn{4}{|l|}{ Duodenum } \\
\hline Bulb & $1.33(0.56)$ & $1.13(0.34)$ & $<0.001$ \\
\hline D2 & $1.28(0.49)$ & $1.17(0.38)$ & 0.10 \\
\hline
\end{tabular}

Abbreviations: NAC, N-acetylcysteine; SD, standard deviation.

Table 3 Percentage of patients with excellent preparation

\begin{tabular}{|l|l|l|l|}
\hline \multicolumn{2}{|l|}{} & $\begin{array}{l}\text { No preparation } \\
(n=106)\end{array}$ & $\begin{array}{l}\text { NAC + simethicone } \\
(n=104)\end{array}$ \\
\hline Esophagus, $n(\%)$ & $63(60)$ & $90(87)$ & $<0.001$ \\
\hline Stomach, $n(\%)$ & $56(53)$ & $93(90)$ & $<0.001$ \\
\hline Fundus & $56(53)$ & $85(82)$ & $<0.001$ \\
\hline Body & $63(60)$ & $91(88)$ & $<0.001$ \\
\hline Antrum & & & $<0.001$ \\
\hline Duodenum, $n(\%)$ & $76(72)$ & $90(87)$ & 0.072 \\
\hline Bulb & $78(74)$ & $86(83)$ & \\
\hline D2 & & \\
\hline
\end{tabular}

Abbreviation: NAC, N-acetylcysteine.

\section{Adverse Events}

No adverse events attributable to the pre-endoscopy drink were seen. All patients tolerated the procedure well and there were no instances of vomiting, aspiration, or intolerance.

\section{Discussion}

This was an investigator blind comparative study of the impact of a pre-endoscopy drink consisting of NAC + simethicone in improving mucosal visibility during routine UGIE in Indian patients at a tertiary high-volume referral center.

As followed routinely in most Indian centers, mandatory fasting for 6 to 8 hours prior to UGIE was followed until recently at our hospital. However, despite periods of recommended fasting, we routinely encountered a lot of air bubbles and mucus impairing mucosal visibility in most of our UGIE procedures. This was especially counter-productive during procedures like NBI and EMR or ESD as fine mucosal characterization is required for delineation and characterization of subtle lesions. ${ }^{14}$

While fasting helps in clearing the food residue from gastroduodenal lumen, it does not help in reduction in air bubbles and mucus. Bubbles are produced due to trapping of gas in the water molecules present in the gastrointestinal lumen. Gas in the gastrointestinal tract is derived either from swallowed air or during fermentation by gut microbiome. Around $20 \mathrm{~mL}$ of gas is present normally in the gastric lumen. ${ }^{15}$
Normally a viscous mucus gel overlies the gastric mucosa that is made up of $95 \%$ water and 5\% cross-linked glycoproteins. ${ }^{16}$ The glycoprotein component keeps the mucus in viscous and fluidic state. With fasting, the glycoprotein content declines, as seen in rat models thus reducing the fluidity of the mucus layer. ${ }^{17}$ This is one of the possible reasons why we often encounter sticky mucus during UGIE in fasting patients. This kind of mucus is often difficult to flush. Use of water alone for flushing mucus has been found ineffective and in fact detrimental for clearance. ${ }^{10}$

Simethicone is a combination of dimethicone (silicone oil) and hydrophobic silica particles. It leads to a reduction in surface tension, surface viscosity, and hydrophobicity at the mucosal surface. ${ }^{18}$ The air bubbles coalesce and disperse easily due to a reduction in surface tension, while the simethicone solution easily disperses along the surface. Simethicone is not absorbed and is virtually inert. It has been routinely advocated for improving the visibility not only for UGIE but also for colonoscopy and video capsule endoscopy. ${ }^{19,20}$ Recently, there has been a concern regarding use of simethicone in the water bottle of flushing pump due to evidence of biofilm formation that may act as a nidus for microorganisms and interfere with endoscope reprocessing. ${ }^{21}$ However, the technique of using simethicone as an orally taken solution is safe and does not damage the endoscope $(s){ }^{22}$ 
NAC has been used as a mucolytic in respiratory diseases since many years. Its mucolytic property has also been used for improved eradication of Helicobacter pylori $i^{23}$ and in softening gastric phytobezoars. ${ }^{24}$ NAC acts by breaking disulfide bonds that stabilize extracellular proteins in the gastric mucus that reduces its thickness and stability, making it more amenable to clearance. ${ }^{25}$

In our study, analysis of the primary objective showed significant improvement in the mucosal visibility during UGIE after administering a pre-endoscopic drink of NAC with simethicone. Excellent preparation with absence of bubbles or mucus was seen in significantly higher percentage of UGIE with NAC + simethicone ( - Fig. 5). These findings are similar to that reported by several other studies on NAC + simethicone in UGIE. $8,9,10,12,26$

There have been some studies that did not find any added advantage of adding NAC to simethicone. ${ }^{27}$ However, we believe use of mucolytic agent offers additional advantage over the defoaming effect of simethicone in clearing the adherent gastric mucus. Most of the endoscopic centers in East Asia, especially in Japan, routinely add a mucolytic (pronase) to simethicone for pre-endoscopy preparation. A recently published meta-analysis reported better total mucosal visibility score when using NAC + simethicone than simethicone alone $(\mathrm{MD}=-0.14(-0.25,-0.03), p=0.01){ }^{28}$

In our study, while significant improvement in mucosal visibility with NAC with simethicone was seen in esophagus, all regions of stomach, and duodenal bulb, insignificant difference was seen in second part of duodenum. This was similarly reported by the studies of Elvas L et $\mathrm{al}^{8}$ and Ahsan et al. ${ }^{29}$ The small amount of preparation in $100 \mathrm{~mL}$ of water may be insufficient to clear the field in distal parts of duodenum. Hence, for video capsule endoscopy and colonoscopy higher volumes of preparation are required to be effective.

The pre-endoscopy drink consisting of NAC with simethicone was easy to prepare and very cheap costing approximately INR 50 per patient preparation. The pre-endoscopy drink was given 10 to 30 minutes prior to UGIE. This is the ideal time period in which UGIE should be undertaken as this conforms to the gastric emptying time for water. Chang et al reported that after 30 minutes there was no difference in the mucosal visibility irrespective of preparation. ${ }^{11}$

There were no adverse events reported with the pre-endoscopy drink and not even a single incidence of aspiration was reported. Similarly, none of the previous studies on pre-endoscopy preparations have documented any major adverse event. This is contrary to perceived risk of aspiration with intake of liquids prior to UGIE that has provoked several institutions and endoscopic societies to maintain strict pre-endoscopy fasting. The ESGE quality improvement measures published in 2016 recommended a 2-hour fasting for liquids and 6-hour fasting for solids prior to UGIE as key performance measure. ${ }^{3}$ However, the more recent guidelines by British Society of Gastroenterology (BSG) and Association of Upper Gastrointestinal Surgeons of Great Britain and Ireland (AUGIS) strongly recommended "mucosal cleansing techniques" for UGIE in their guidelines published in 2017. ${ }^{30}$
We thus present a simple technique of improving the mucosal visibility by using a pre-endoscopic drink of NAC + simethicone. To the best of our knowledge, this is the first Indian study evaluating the outcomes of a simple pre-endoscopy preparation. It is easily available, cheap, convenient to administer, well tolerated, and very effective. Shifting from conventional unprepared UGIE to adequately prepared UGIE is the need of the hour, especially in India, now that we are in steadily incorporating advanced diagnostic and therapeutic procedures like NBI, EMR, and ESD in most of our endoscopy centers.

Not only does this preparation improve mucosal visibility, but also increases lesion detection rate, ${ }^{8}$ it also cuts down on the operator time and reduces the need for frequent and sustained water flush. ${ }^{9}$ These are also useful attributes as they could optimize the efficiency of most of our endoscopy units that are understaffed and overburdened. Water flushing alone is usually counterproductive as it only spreads the mucus over a wider area without dissolving it. Time and effort saved in clearing the endoscopic field by frequent flushing could rather be utilized for improved lesion detection and careful assessment.

Our study has a few drawbacks. We could not compare the mucosal visibility in every individual patient prior to premedication to assess the impact of individual preparations. Though it was a retrospective study, we took an adequately matched control group. The endoscopists were not blinded to the preparation given, but this was unlikely to affect the study outcome as the only job of the endoscopist was to take standard images as per departmental protocol prior to use of water flush. The investigator who analyzed the images was blinded and this was the most important part. The time difference due to preparation was not studied as it was difficult to accurately measure accurate endoscopy time in busy schedule. Moreover, time taken for UGIE would also differ between operators and indications; hence, it might not be an ideal representation.

In conclusion, we would recommend a pre-endoscopy drink of NAC with simethicone prior to all elective UGIE procedures. The study suggests usefulness of NAC with simethicone in improving mucosal visibility and warrants further investigation for effectiveness in a prospective trial and eventual application as a standard of care.

\section{Conflict of Interest}

None.

\section{References}

1 Kastenberg D, Bertiger G, Brogadir S. Bowel preparation quality scales for colonoscopy. World J Gastroenterol 2018; 24(26):2833-2843

2 Faigel DO, Eisen GM, Baron TH, et al; Standards of Practice Committee. American Society for Gastrointestinal Endoscopy. Preparation of patients for GI endoscopy. Gastrointest Endosc 2003;57(4):446-450

3 Bisschops R, Areia M, Coron E, et al. Performance measures for upper gastrointestinal endoscopy: a European Society of Gastrointestinal Endoscopy (ESGE) Quality Improvement Initiative. Endoscopy 2016;48(9):843-864 
4 Gotoda T, Uedo N, Yoshinaga S, et al. Basic principles and practice of gastric cancer screening using high-definition whitelight gastroscopy: eyes can only see what the brain knows. Dig Endosc 2016;28(Suppl 1) :2-15

5 Chen H-W, Hsu H-C, Hsieh T-Y, Yeh M-K, Chang W-K. Premedication to improve esophagogastroduodenoscopic visibility: a meta-analysis and systemic review. Hepatogastroenterology 2014;61(134):1642-1648

6 Tongprasert S, Sobhonslidsuk A, Rattanasiri S. Improving quality of colonoscopy by adding simethicone to sodium phosphate bowel preparation. World J Gastroenterol 2009; 15(24):3032-3037

7 Lee GJ, Park SJ, Kim SJ, Kim HH, Park MI, Moon W. Effectiveness of premedication with Pronase for visualization of the mucosa during endoscopy: a randomized, controlled trial. Clin Endosc 2012;45(2):161-164

8 Elvas L, Areia M, Brito D, Alves S, Saraiva S, Cadime AT. Premedication with simethicone and $\mathrm{N}$-acetylcysteine in improving visibility during upper endoscopy: a double-blind randomized trial. Endoscopy 2017;49(2):139-145

9 Neale JR, James S, Callaghan J, Patel P. Premedication with $\mathrm{N}$-acetylcysteine and simethicone improves mucosal visualization during gastroscopy: a randomized, controlled, endoscopist-blinded study. Eur J Gastroenterol Hepatol 2013;25(7):778-783

10 Monrroy H, Vargas JI, Glasinovic E, et al. Use of N-acetylcysteine plus simethicone to improve mucosal visibility during upper GI endoscopy: a double-blind, randomized controlled trial. Gastrointest Endosc 2018;87(4):986-993

11 Chang W-K, Yeh M-K, Hsu H-C, Chen H-W, Hu M-K. Efficacy of simethicone and $\mathrm{N}$-acetylcysteine as premedication in improving visibility during upper endoscopy. J Gastroenterol Hepatol 2014;29(4):769-774

12 A randomized controlled trial of pre-procedure simethicone and $\mathrm{N}$-acetylcysteine to improve mucosal visibility during gastroscopy - NICEVIS. - - NCBI [Internet]. [cited 2020 Apr 19]. Availablefrom:https://www.ncbi.nlm.nih.gov/pubmed/?term=A+ randomized+controlled+trial+of+pre-procedure+simethicone+ and $+\mathrm{N}$-acetylcysteine+to+improve+mucosal+visibility+during+gastroscopy+\%E2\%80\%93+NICEVIS. Accessed December 8, 2020

13 Veeraraghavan K, Abel J, Shreyas V, et al. Efficacy of Simethicone and n-acetylcysteine combination premedication prior to esophagogastroduodenoscopy to improve mucosal visibility - a prospective double blinded randomized placebo controlled trial (CTRI/2017/09/009913). In: Endoscopy [Internet]. Georg Thieme Verlag KG; 2018 [cited 2020 Apr 25]. p. OP212. Available from: http://www.thieme-connect.de/DOI/ DOI?10.1055/s-0038-1637249. Accessed December 8, 2020

14 Ali S, Zhou F, Braden B, et al. An objective comparison of detection and segmentation algorithms for artefacts in clinical endoscopy. Sci Rep 2020;10(1):2748

15 The pathogenesis of bloating and visible distension in irritable bowel syndrome. - - NCBI [Internet]. [cited 2020 Apr 21]. Available from: https://www.ncbi.nlm.nih.gov/ pubmed/15862934. Accessed December 8, 2020

16 Corfield AP, Carroll D, Myerscough N, Probert CS. Mucins in the gastrointestinal tract in health and disease. Front Biosci 2001;6:D1321-D1357

17 Kinoshita M, Igarashi S, Kume E, Saito N, Arakawa K. Fasting induces impairment of gastric mucosal integrity in non-insulin-dependent diabetic $(\mathrm{db} / \mathrm{db})$ mice. Aliment Pharmacol Ther 2000;14(3):359-366

18 Brecević L, Bosan-Kilibarda I, Strajnar F. Mechanism of antifoaming action of simethicone. J Appl Toxicol 1994; 14(3):207-211

19 Song HJ. [Optimal bowel preparation for capsule endoscopy and device-assisted enteroscopy]. Korean J Gastroenterol 2020;75(2):74-78

20 Hassan C, East J, Radaelli F, et al. Bowel preparation for colonoscopy: European Society of Gastrointestinal Endoscopy (ESGE) Guideline - update 2019. Endoscopy 2019;51(8):775-794

21 Simethicone residue remains inside gastrointestinal endoscopes despite reprocessing. - - NCBI [Internet]. [cited 2020 Apr 21]. Available from: https://www.ncbi.nlm.nih.gov/pubmed/?ter$\mathrm{m}=$ simethicone+residue+remains+inside+despite+endoscope+ reprocessing. Accessed December 8, 2020

22 Simethicone use during gastrointestinal endoscopy: Position statement of the Gastroenterological Society of Australia Devereaux - 2019 - Journal of Gastroenterology and Hepatology WileyOnlineLibrary[Internet].[cited2020Apr21].Availablefrom: https://onlinelibrary.wiley.com/doi/full/10.1111/jgh.14757. Accessed December 8, 2020

$23 \mathrm{~N}$-acetylcysteine as an adjuvant therapy for Helicobacter pylori eradication. - - NCBI [Internet]. [cited 2020 Apr 21]. Available from: https://www.ncbi.nlm.nih.gov/pubmed/30746681. Accessed December 8, 2020

24 Endoscopic and enzymatic treatment of gastric bezoar with acetylcysteine. - - NCBI [Internet]. [cited 2020 Apr 21]. Available from: https://www.ncbi.nlm.nih.gov/pubmed/?term=Endoscopic+and+enzymatic+treatment+of+gastric+bezoar+with+acetylcysteine.+Endoscopy+2002. Accessed December 8, 2020

25 The add-on $\mathrm{N}$-acetylcysteine is more effective than dimethicone alone to eliminate mucus during narrow-band imaging endoscopy: a double-blind, rando... - - NCBI [Internet]. [cited 2020 Apr 21]. Available from: https://www.ncbi.nlm.nih.gov/pubmed/?term= The+add-on+N-acetylcysteine+is+more+effective+than+dimet hicone+alone+to+eliminate+mucus+during+narrow-band+imaging+endoscopy $\% 3 \mathrm{~A}+\mathrm{a}+$ double-blind $\% 2 \mathrm{C}+$ randomized+controlled+trial. Accessed December 8, 2020

26 Mahawongkajit P, Kanlerd A. A prospective randomized controlled trial comparing simethicone, $\mathrm{N}$-acetylcysteine, sodium bicarbonate and peppermint for visualization in upper gastrointestinal endoscopy. Surg Endosc 2020

27 Asl SMKH, Sivandzadeh GR. Efficacy of premedication with activated Dimethicone or $\mathrm{N}$-acetylcysteine in improving visibility during upper endoscopy. World J Gastroenterol 2011;17(37):4213-4217

28 Li Y, Du F, Fu D. The effect of using simethicone with or without $\mathrm{N}$-acetylcysteine before gastroscopy: a meta-analysis and systemic review. Saudi J Gastroenterol 2019;25(4):218-228

29 Ahsan M, Babaei L, Gholamrezaei A, Emami MH. Simethicone for the preparation before esophagogastroduodenoscopy. Diagn Ther Endosc 2011;2011:484532

30 Beg S, Ragunath K, Wyman A, et al. Quality standards in upper gastrointestinal endoscopy: a position statement of the British Society of Gastroenterology (BSG) and Association of Upper Gastrointestinal Surgeons of Great Britain and Ireland (AUGIS) Gut 2017;66(11):1886-1899 